Please do not remove this page

RMIT

UNIVERSITY

\title{
Bias-free phase tracking with linear and nonlinear systems
}

Sithamparanathan, Kandeepan; Evans, R

https://researchrepository.rmit.edu.au/esploro/outputs/9921858211401341/filesAndLinks?institution=61RMIT_INST\&index=null

Sithamparanathan, K., \& Evans, R. (2010). Bias-free phase tracking with linear and nonlinear systems. IEEE Transaction on Wireless Communications, 9(12), 3779-3789.

https://doi.org/10.1109/TWC.2010.100510.100107

Document Version: Submitted Version

Published Version: https://doi.org/10.1109/TWC.2010.100510.100107

Repository homepage: https://researchrepository.rmit.edu.au

(C) 2010 IEEE

Downloaded On 2023/04/26 20:16:23 +1000

Please do not remove this page 
Thank you for downloading this document from the RMIT Research Repository.

The RMIT Research Repository is an open access database showcasing the research outputs of RMIT University researchers.

RMIT Research Repository: http://researchbank.rmit.edu.au/

\section{Citation:}

Sithamparanathan, K and Evans, R 2010, 'Bias-free phase tracking with linear and nonlinear systems', IEEE Transaction on Wireless Communications, vol. 9, no. 12, pp. 3779-3789.

See this record in the RMIT Research Repository at:

https://researchbank.rmit.edu.au/view/rmit:23510

Version: Submitted Version

Copyright Statement: (C) 2010 IEEE

Link to Published Version:

http://dx.doi.org/10.1109/TWC.2010.100510.100107 
(c) (c) 2010 IEEE. Personal use of this material is permitted. Permission from IEEE must be obtained for all other uses, in any current or future media, including reprinting/republishing this material for advertising or promotional purposes, creating new collective works, for resale or redistribution to servers or lists, or reuse of any copyrighted component of this work in other works. 


\title{
Bias-Free Phase Tracking with Linear and Nonlinear Systems
}

\author{
Sithamparanathan Kandeepan Senior Member IEEE, Rob Evans Fellow IEEE
}

\begin{abstract}
The arctan function is a well-known Maximum Likelihood (ML) estimator of the phase angle $\alpha \in$ $[-\pi, \pi)$ of a complex signal in additive white Gaussian noise. In this paper we revisit the arctan-based ML phase estimator and identify the bias problem for phase tracking. We show that the posteriori probability density function of $\alpha$ becomes a bi-modal distribution for small values of signal to noise ratio $\rho$ and larger values of $\alpha$. In such cases the mean and the mode differ from each other, and as a result when such ML phase estimates are used as an input to a linear system (LS), example for phase tracking, the resulting output (which is essentially the mean value of the phase) differs from its true value which is the mode. In such situations there exist a mean (tracking) error at the output of the LS from its true value $\alpha$, and in (non-Bayesian) statistical terms there exist a bias in the estimates. In this paper, we provide some statistical analysis to explain the above problem, and also provide solutions for bias correction when a LS is used for tracking phase. Furthermore, we also provide two nonlinear phase tracking systems, 1) a Monte-Carlo based sequential phase tracking technique and 2) a second-order digital-phase locked loop based method, for bias-free phase tracking which eliminate the bias problem that occurs in the case of linear phase tracking with ML estimates.
\end{abstract}

\section{Index Terms}

phase estimation, phase tracking, phase bias-correction, bias-free phase tracking, arctan function

\section{INTRODUCTION}

Synchronization of carrier and phase is a well know problem in communications [28],[46]-[58], in this paper we address the phase estimation problem. The problem of phase estimation is well treated in the field of signal processing for various applications. In the fields of radar, sonar and communications etc. phase estimation and tracking is performed to predict, recover or decode information, and especially in the recent years it is also used in cognitive radios for detecting legacy users of the spectrum. In almost every application the phase is required to be estimated under noisy conditions, for example signals received with additive noise and multiplicative noise (fading) in certain scenarios. Using the noise statistics we formulate the phase estimation problem as a statistical parameter estimation problem and use the existing estimation techniques [1] to find suitable solutions for different application clusters. In this paper we consider phase estimation of a complex signal corrupted with additive white Gaussian noise (AWGN). This topic has been treated extensively in the literature and one could find many papers in various applications. Here, we revisit this topic again considering the bias problem associated with phase tracking based on the arctan Maximum

Manuscript submitted to the IEEE Transactions on Wireless Communications for review on 26 Jan 2010. The authors would like to thank the European Commission for funding the project under the Framework Program - 7 (EU-FP7) in the Information and Communications Technology cluster. EUWB (FP7-ICT-215669). The authors would also like to thank the National ICT Australia (NICTA) for partly funding the research during the early stages. 
Likelihood (ML) estimates, and provide solutions for bias correction. For phase estimation with AWGN, the ML estimation technique is proven to be the optimal technique which is also a well presented subject in the literature [1]-[11]. The nonlinear arctan function is used in such ML phase estimators in order to map the signal phase to its corresponding four quadrant phase plane. We present some statistical analysis on the performance of the nonlinear arctan function when used as a ML phase estimator and point out a particular problem, a bias problem, associated with it especially observed at low signal to noise ratio (SNR) levels. This problem was studied by Lovell and Williamson in [12] and subsequently by Clarkson, Kootsookos and Quinn in [13] for frequency estimation based on phase angle estimates, and later by Peters and Kay in [2] for the estimation of phase. In [2], the authors analyze the bias in phase estimates by considering the eigenvalues of the smoothing kernel for the arctan-based maximum likelihood estimator (ML) for the phase. However there were no solutions provided to solve the bias problem in the above studies.

In our work we analyze the problem differently to [2], [12] and [13], and provide solutions for bias correction for linear phase tracking. Furthermore, we present two nonlinear tracking techniques, apart from the linear techniques, 1) based on sequential Monte-Carlo method which is a statistical mode estimation method and 2) based on arctan-based Digital Phase Locked Loops (D-PLL) technique [14]-[33]. The two proposed nonlinear methods eliminate the bias problem that exists in linear phase tracking. In the literature, the Bayesian approach for phase tracking namely particle-filter-based sequential Monte Carlo tracking techniques [35] exist for tracking Wiener phase [36]-[39] by treating the phase noise as Gaussian. In our work however, we use the true posteriori distribution of the phase for tracking by providing an importance function derived from the posteriori density function. The second nonlinear technique D-PLL on the other hand is a well-known method for phase tracking and our contribution comes in here by presenting the D-PLL as a bias-free phase tracking technique a topic which has not been treated so far in the literature of PLL or D-PLL. Note that the arctan-based D-PLL which we consider in this paper [26], [28] is generally referred to as the tan-lock loop in literature [15]-[18], whereas the traditional D-PLL/PLL on the other hand has a 'sine' type of phase detector [14], [30], [31].

Now, we briefly summarize some of the fundamental work performed in phase estimation whilst noting that there exist numerous papers on this topic depending on the application cluster. Hence, we only consider the references closely related to our work in this paper. Phase estimation and tracking can be performed using feed forward or feedback techniques depending on the requirement and the application. Feed forward techniques are based on block estimates for $N$ number of samples per block with a given SNR. The authors in [3] present a maximum a-posteriori (MAP) phase estimator based on the arctan function including 
an extensive analysis on its performance and a brief comparison against the feedback phase estimator. Viterbi and Viterbi [6] presented a nonlinear phase estimation technique for phase shift keyed (PSK) communications using burst transmissions considering the modulation effects on the received signal, their work was then followed and referenced by many other researchers and the performances were compared with other estimation techniques, namely [7] and [8]. Rife and Boostyn presented a maximum likelihood (ML) based technique for phase estimation and compared the performance with the Cramer-Rao bound (CRB) on its estimation error variance [4]. Phase estimation in fading channels is treated in [5] considering the minimum mean square error (MMSE) estimator. Recently, authors in [11] have presented a MAP/ML phase estimation technique by assuming the a-priori distribution of the phase to be a Tikhonov distribution. In all of the above mentioned feed forward techniques the arctan function is used to map the phase of a complex signal on to its four-quadrant phase plane in one way or the other, which gives rise to the bias problem that relates to the work presented in this paper.

The analog and the digital phase-locked loops (PLL and DPLL) are the most commonly used feedback techniques [14]-[33] to acquire and track phase. Kalman filters [34] on the other hand are also feedback loops used to track phase with the arctan-based ML estimates. PLL and DPLL have been very well treated in literature for applications such as communications, radar and sonar etc. Viterbi [31], Gardner [14] and Gupta [33] have presented some of the most fundamental work on analog PLL and the related performance analysis. Lindsey and Chie [20] have presented some fundamental work on DPLL similar to the analog PLL. As mentioned before, the feedback loop that utilizes the arctan function is known as the Tanlock loop (TLL) [15]-[18]. Tanlock loops are known to have better tracking capabilities with better linear range compared to the PLL or the DPLL. During the analog era, the arctan function was represented by a sawtooth phase detector within the loop which emulates the functional characteristics of the mathematical arctan function [21]-[23]. Moreover, the authors in [40] propose and iterative decoding method to detect signals corrupted with channel phase noise by modeling it as a Tikonov distribution. Again, none of the work above on feedback phase estimation addresses the bias issue considered in this paper.

Another important phase tracking technique to be mentioned here is the vector tracking method [41],[42]. Vector tracking is considered to be one of the best methods to correct phase errors of a complex vector without the need to estimate the phase. Since vector tracking does not require to estimate the phase it does not suffer from the bias issue addressed in this paper. The phase-bias addressed in this paper is generated by the nonlinear $\arctan$ function when estimating the phase $\alpha$ from a vector $\exp (j \alpha)$, and since the vector tracking technique does not involve the arctan function it does not suffer from the particular bias. However, 
when the arctan function is used to estimate the phase $\alpha$ of a vector $\exp (j \alpha)$ which had been obtained by means of vector tracking it will still suffer the bias problem mentioned in this paper at low signal to noise ratio and for higher values of $\alpha$, which we explain in the subsequent sections. It should also be noted here that phase correction using vector tracking solves the problem of phase-bias for example in communications for detecting data sequence etc [41],[42]. However, in this paper we are addressing the applications which require the estimation of the phase not only for communications but applications such as radar, localization, sonar, bearing only estimation, deep space science etc where ever phase information is required for noisy inferences.

The rest of the paper is organized as follows. In Section II we introduce the ML phase estimator, in Section III we perform statistical analysis on the arctan-based ML estimator explaining the bias problem associated with it. In Section IV we analyze the bias problem for linear systems (LS), and in Section V we provide solutions for bias correction followed by some numerical examples in Section VI. In Sections VII and VIII we provide nonlinear phase tracking techniques for bias-free phase estimation, and finally we draw some concluding remarks in Section IX.

\section{ML Phase Estimation}

In this section we present the ML estimator based on the $\arctan$ function $\arg \{$.$\} for the phase of a$ complex signal in AWGN. Consider a complex discrete signal given by

$$
r[n]=A \exp (j \Omega n+j \alpha)+\eta[n]
$$

where $\Omega$ is a known parameter, $\alpha \in[-\pi, \pi)$ is the deterministic phase to be estimated assumed to be a constant within the duration of consideration but changes over time, $A$ is the signal amplitude assumed to be a constant (or a slowly varying parameter), and $\eta[n]$ is the complex Gaussian noise process with zero mean and a variance of $2 \sigma^{2}$ expressed as $\eta \sim C N\left(0,2 \sigma^{2}\right)$. For convenience we omit the time index-n in the equations hereafter unless it is required. The ML estimator for phase $\alpha$ [1],[4] is then given by maximising the likelihood function $p(r \mid \alpha)$

$$
\theta=\arg \max _{\alpha}\{p(r \mid \alpha)\}
$$

Since the a-priori density of $\alpha$ is given by $p(\alpha)=1 / 2 \pi$ for $-\pi \leq \alpha<\pi$, the ML estimator can also be equivalently written as the MAP estimator by maximizing the posteriori density $p(\alpha \mid r)$ given by,

$$
\theta=\arg \max _{\alpha}\{p(\alpha \mid r)\}
$$


Then, knowing that the noise distribution is Gaussian and provided that $\Omega$ is perfectly known to the receiver, the ML phase estimator [4], [29], [30] becomes

$$
\theta=\arg \left\{\sum_{n=1}^{N} r \exp (-j \Omega n)\right\}
$$

where $N$ is the number of samples used per estimate and $\arg \{$.$\} is the \arctan$ function that maps the phase of the complex signal to its corresponding four-quadrant phase plane. The estimator in (4) is a well-known estimator in literature, and is also known as the MAP and the MMSE estimators [1], [5]. In the following sections we study the distribution and the statistical properties of the phase estimates $\theta$ and identify the bias problem associated with the ML estimator in (4) especially at low SNR and for higher values of $\alpha$.

\section{Statistical Properties of the Phase Estimate}

The distribution and the statistical properties of the ML phase estimates are presented in this section. The density of $\theta$ conditioned on the received signal to noise power ratio $\rho$ and the phase angle $\alpha$ is given by the function $f_{\theta}(\theta \mid \rho, \alpha)$ for the range of $-\pi<\theta<\pi$ as given in equation (5). [Note that the function in (5) can be found in many references in different forms for example in [43]-[45].]

$$
\begin{gathered}
f_{\theta}(\theta \mid \rho, \alpha)= \begin{cases}\exp (-\varsigma / 2)\left[\frac{1}{2 \pi}-\exp \left(\frac{\Gamma}{2}\right)\left(\frac{\Gamma}{2 \pi}\right)^{\frac{1}{2}} Q(\sqrt{\Gamma})\right] & \text { for } \frac{\pi}{2}<\theta \leq \pi \\
\exp (-\varsigma / 2)\left[\frac{1}{2 \pi}+\exp \left(\frac{\Gamma}{2}\right)\left(\frac{\Gamma}{2 \pi}\right)^{\frac{1}{2}}(1-Q(\sqrt{\Gamma}))\right] & \text { for }-\frac{\pi}{2}<\theta \leq \frac{\pi}{2} \\
\exp (-\varsigma / 2)\left[\frac{1}{2 \pi}-\exp \left(\frac{\Gamma}{2}\right)\left(\frac{\Gamma}{2 \pi}\right)^{\frac{1}{2}} Q(\sqrt{\Gamma})\right] & \text { for }-\pi<\theta \leq-\frac{\pi}{2}\end{cases} \\
\text { where }, \varsigma=2 N \rho, \Gamma=\varsigma \cos ^{2}(\theta-\alpha) \text { and }, Q(x)=\frac{1}{2 \pi} \int_{x}^{\infty} \exp \left(-u^{2} / 2\right) d u
\end{gathered}
$$

The signal to noise ratio ${ }^{1}$ given by $\rho=(E[A])^{2} /\left(2 \sigma^{2}\right)$ expressed in (6) is directly measured on the received signal $r$. In (5) three different regions for $\theta$ are observed due to the discontinuity of the arctan function at points $-\pi / 2$ and $\pi / 2$ between $-\pi$ and $\pi$. Note that, when $N=1, f_{\theta}(\theta \mid \rho, \alpha)$ becomes the posteriori density function $p(\alpha \mid r)$ of the phase $\alpha$ in standard statistical terms. From (5) we observe that $f_{\theta}(\theta \mid \rho, \alpha)$ is maximized when $\theta=\alpha$, and therefore we verify that the estimator in (4) is indeed a ML/MAP estimator. We also observe that when there is no signal present or when the noise power is infinite the distribution becomes a uniform distribution given by $f_{\theta}(\theta \mid \rho, \alpha)=1 /(2 \pi)$, for $\theta \in[-\pi, \pi)$. Further, we see that the density becomes less peaky and flat when $\varsigma$ is reduced, and hence it raises its tail and becomes a heavy tailed distribution. When $|\alpha|$ is increased the density becomes asymmetric, and when $\varsigma$ is reduced

\footnotetext{
${ }^{1}$ The signal to noise ratio $\rho$ is the actual received SNR, whereas $\varsigma$ is the effective SNR based on the number of samples $N$, in this paper we use both to measure the performances of the system. Further, when it comes to the estimation of SNR in the section to follow, we only talk about estimating $\rho$.
} 
at the same time it becomes a bi-modal distribution. Figure 1 shows the probability density function of $\theta$ conditioned on $\rho$ for various values of $\alpha$ and $\varsigma$. The rising of the tail is observed in the figure when the signal to noise ratio becomes low and also when the input angle becomes large. We also depict some simulation results to verify the analytical expressions given in (5) and (6). The simulations were performed using Monte-Carlo methods with $\alpha=45^{\circ}$ for two SNR values of $\varsigma=0 \mathrm{~dB}$ and $\varsigma=-10 \mathrm{~dB}$, and also for $\alpha=125^{\circ}$ with $\varsigma=-5 \mathrm{~dB}$.

A key point to observe from the figure depicting the density curve of the phase estimates is the rising of the tail at the furthest end to $\alpha$ on the $\mathrm{x}$-axis. Because of this single-sided rising of the tail the distribution becomes a bimodal distribution where the mean and the mode differ from each other. By observing such variations in the distribution it is easy to identify that the estimates in (4) is actually the sample mode of $\theta$ but not the expected value of $\theta$. Therefore, when the ML phase estimates are passed through a linear system such as a linear filter, the output of the filter differs from the true phase value $\alpha$ introducing a bias. This is because the filter provides the expected value of $\theta$ instead of the mode of $\theta$. In the rest of the sections we provide bias correction techniques for linear phase tracking and nonlinear bias free tracking techniques and compare the relative performance.

\section{A. Characteristic Curve and Phase Estimator Gain}

The characteristic curves of the phase estimator are given by the expected values of the phase estimates $\theta$ plotted against all possible values of the input phase for various effective signal to noise ratio values ५. The idea of such characteristic curves for phase estimates was adopted from the PLL literature [23], [30] (and well before the time of [23]) which was originally in the form of phase detector characteristics or the S-curves. The noise free characteristic of the phase estimator is a ramp function with unit gradient given by $g(\beta) \triangleq \beta$ for the range of $-\pi \leq \beta<\pi$. In the presence of noise though the characteristics of the phase estimator changes. The noisy phase estimator can be modeled using $g(\theta)$ as

$$
\theta=g\left(\beta+\theta_{v}\right)
$$

where $\theta_{v}$ is the phase noise having a density function described by (5) with zero mean (i.e. $\alpha=0$ ), and the noisy characteristics of the phase estimator is then given by taking the expectation of (7), defined by

$$
g_{v}(\theta \mid \beta) \triangleq \int_{-\pi}^{\pi} g\left(\beta+\theta_{v}\right) f_{\theta_{v}}\left(\theta_{v}\right) d \theta_{v}
$$


where the density function of $\theta_{v}$ is given by $f_{\theta_{v}}\left(\theta_{v}\right)=f_{\theta}(\theta \mid \rho, \alpha=0)$. The integration in (8) is computed after expanding $g\left(\beta+\theta_{v}\right)$ using the Fourier series coefficients and using the fact that $\theta_{v}$ is zero mean. Appendix 1 provides the solution to the integration (derived from [23]) giving us a closed form expression for the characteristics function given by

$$
g_{v}(\theta \mid \beta)=\sqrt{\pi \varsigma} \exp \left(-\frac{\varsigma}{2}\right) \sum_{k=1}^{\infty} \frac{(-1)^{k+1}}{k}\left[I_{\frac{k-1}{2}}\left(\frac{\varsigma}{2}\right)+I_{\frac{k+1}{2}}\left(\frac{\varsigma}{2}\right)\right] \sin (k \beta)
$$

where $I_{x}($.$) is the modified Bessel function of order x$. Figure 2 depicts the characteristic curves for the ML phase estimator. The curves show the differences between the expected values of the estimates and the true input values of the phase for $\beta=\alpha$. The expected values differ greatly from the true values of the phase as we observe (in other words the bias increases), when $\varsigma$ is reduced and $\alpha$ is increased. The phase estimator gain $K_{p}$ is also a term commonly used in the PLL literature to describe the phase detector gain of the loop [14], [30]. The phase estimator gain is defined by the slope (first derivative) of $g_{v}(\theta \mid \beta)$ at the point of origin which varies with the signal to noise ratio. Mathematically it is given by

$$
\left.K_{p} \triangleq \frac{d\left[g_{v}(\theta \mid \beta)\right]}{d \beta}\right|_{\beta}=0
$$

By substituting (9) in (10), we directly find a closed form expression for $K_{p}$, given by

$$
K_{p}=\sqrt{\pi \varsigma} \exp \left(-\frac{\varsigma}{2}\right) \sum_{k=1}^{\infty}(-1)^{k+1}\left[I_{\frac{k-1}{2}}\left(\frac{\varsigma}{2}\right)+I_{\frac{k+1}{2}}\left(\frac{\varsigma}{2}\right)\right]
$$

The phase estimator gain is plotted in Figure 3 with respect to $\varsigma$. Ideally, the phase estimator gain is expected to be unity or a constant when the signal to noise ratio is changed, but from the figure we observe that the phase estimator gain reduces when $\varsigma$ is reduced, which is an undesirable feature of the estimator. In the successive sections we use the results presented in this section to describe the bias problem in phase tracking with linear systems and provide solutions for bias correction.

\section{B. Mean Squared Error of the Estimates}

The Mean Squared Error (MSE) of the estimates is derived similar to the characteristics equation. First we compute the non-central second moment of the phase estimates $\theta$ and then use (9) to calculate the MSE. The phase estimator model in (7) is used to compute the non-central second moment of the estimated phase given by

$$
E\left[\theta^{2}\right]=\int_{-\pi}^{\pi} g^{2}\left(\beta+\theta_{v}\right) f_{\theta_{v}}\left(\theta_{v}\right) d\left(\theta_{v}\right)
$$


We expand $g^{2}\left(\beta+\theta_{v}\right)$ using the Fourier series coefficients before performing the integration in (12). The solution to the integral is given in Appendix 2, and the final expression for the non-central second moment of $\theta$ is given by

$$
E\left[\theta^{2}\right]=\frac{\pi^{2}}{3}+2 \sqrt{\pi \varsigma} \exp \left(-\frac{\varsigma}{2}\right) \sum_{k=1}^{\infty} \frac{(-1)^{k}}{k^{2}}\left[I_{\frac{k-1}{2}}\left(\frac{\varsigma}{2}\right)+I_{\frac{k+1}{2}}\left(\frac{\varsigma}{2}\right)\right] \cos (k \beta)
$$

where $I_{x}($.$) is the modified Bessel function of order x$. From (13) and (9), the mean squared error (MSE) is then given by

$$
\sigma_{M S E}^{2}(\theta \mid \beta)=E\left[\theta^{2}\right]-g_{v}^{2}(\theta \mid \beta)
$$

We see that the MSE of the phase estimates depends on the input phase angle $\alpha$. Furthermore, it reaches a value of $\pi^{2} / 3$ at low signal to noise ratio values due to the fact that $f_{\theta}(\theta \mid \rho)$ becomes a uniform distribution of $1 / 2 \pi$ between $[-\pi, \pi)$ for low values of $\varsigma$.

\section{Linear Phase Tracking}

In the previous section we saw that the phase estimates produced by the ML estimator is equivalent to the mode of the conditional posteriori density function $f_{\theta}(\theta \mid \rho, \alpha)$. It was also observed that the mean and the mode values of $f_{\theta}(\theta \mid \rho, \alpha)$ differ from each other when the signal to noise ratio becomes low and the phase value becomes large. Considering these two observations, in this section, we further describe the bias problem observed in phase tracking using linear systems. Figure 4 depicts the block diagram of the linear phase tracking/estimation model that we adopt. Considering the phase estimation/tracking problem for the signal $r[n]$ given in (1), the discrete output $\varphi_{f}[n]$ of the filter with a given impulse response $h[m]$ and input $\theta[n]$ as depicted in Figure 4 is given by

$$
\varphi_{f}[n]=\sum_{m=0}^{M_{F}-1} h[m] \theta[n-m]
$$

where $M_{F}$ is the length of the filter. By directly taking the expectation with respect to time on either sides of (15), we obtain,

$$
E\left[\varphi_{f}[m]\right]=K_{0} E[\theta[m]]
$$

where $K_{0}$ is the filter gain. Then by using (8) in (16), we have,

$$
E\left[\varphi_{f}[m]\right]=K_{0} g_{v}(\theta \mid \alpha)
$$

From (17) we see that the expected value of the output of the filter is proportional to the expected value of the ML phase estimates $E[\theta[m]]$, and hence there exist a bias in $\varphi_{f}[m]$ due to the bias in $E[\theta[m]]$. 
The bias is observed especially for low levels of $\varsigma(<6 \mathrm{~dB})$ and for higher values of $\alpha$, note that lower values of $\varsigma$ is obtained when $N$ or $\rho$ is small. A similar argument to the above also can be extended to an IIR filter, or any linear systems in general, and hence we generalize the bias problem for any linear phase tracking/estimation system.

\section{Bias Correction for Linear Phase Tracking}

In this section we present some techniques to correct the bias in the phase as described in the previous section for phase estimation using linear systems. We present two techniques which can be conveniently used for real time applications with varying levels of complexity. The basic idea behind the bias correction techniques that we present is to simply map $g_{v}(\theta \mid \alpha)$ (that is the phase estimates $\varphi_{f}$ ) to its corresponding values of $\alpha$ (true phase input) based on the functional expression in (9). From Figure 2 we observe that the functional mapping given by $\alpha \rightarrow g_{v}(\theta \mid \alpha)$ defined by $D^{\alpha} \rightarrow D^{g}: \alpha \mapsto g_{v}(\theta \mid \alpha)$, is a non-injective and a surjective mapping, where the domains $D^{\alpha}$ and $D^{g}$ are theoretically defined by $\forall d^{\alpha} \in D^{\alpha},-\pi \leq d^{\alpha}<\pi$ and $\forall d^{g} \in D^{g},-\pi \leq d^{g}<\pi$. We further note that the functional mapping of $g_{v}^{-1}(\theta \mid \alpha)$ defined by $D^{g} \rightarrow D^{\alpha}: g_{v}(\theta \mid \alpha) \mapsto \alpha$ has two solutions for $\alpha$. In the following sections we present some techniques to find $g_{v}^{-1}(\theta \mid \alpha)$ for bias correction in linear phase tracking.

\section{A. Technique-1: Linear Approximation of $g_{v}(\theta \mid \alpha)$}

The first technique for bias correction approximates the expression in (9) to find a reasonable solution for the inverse of $g_{v}(\theta \mid \alpha)$. The characteristic function in (9) is approximated by considering small angle approximation given by $\sin (k \alpha) \approx k \alpha$. Then from (9), we have,

$$
g_{v}(\theta \mid \alpha)=\alpha \sum_{k=1}^{\infty}(-1)^{k+1} \sqrt{\pi \varsigma} \exp \left(-\frac{\varsigma}{2}\right)\left[I_{\frac{k-1}{2}}\left(\frac{\varsigma}{2}\right)+I_{\frac{k+1}{2}}\left(\frac{\varsigma}{2}\right)\right]
$$

Using (11) we can rewrite (18) in terms of the phase estimator gain $K_{p}$, and therefore the linear approximation directly gives us,

$$
\alpha=\frac{g_{v}(\theta \mid \alpha)}{K_{p}}
$$

Using the phase estimator gain $K_{p}$ described in section-III we propose a new phase tracking method with bias correction for the ML arctan-based phase estimates involving linear systems. The block diagram of the phase tracker with bias correction is depicted in Figure 5. In the new method, in addition to the phase tracking blocks depicted in Figure 4, we also perform a signal to noise ratio estimation at the receiver over $N$ samples to compute the corresponding phase estimator gain $K_{p}$. In Figure 5, the function $\Gamma_{\theta}$ represents 
the mapping of $\Gamma: g_{v}(\theta \mid \alpha) \mapsto \alpha$, and which in this case the output of the linear system $\varphi_{f}$ is scaled by $1 / K_{p}$ to generate the new phase value $\varphi$. The error in the signal to noise ratio estimate will degrade the accuracy of $\varphi$. The computation of the phase estimator gain $K_{p}$ can be performed in two ways, 1) computing $K_{p}$ using the closed form expression as in (11), and 2) by maintaining a look-up table with pre-computed values of $K_{p}$ and mapping the corresponding value based on the estimate of $\rho$ for a given $N$. Multiplying by $1 / K_{p}$ also increases the noise variance since $K_{p} \leq 1$ especially when the SNR is reduced.

\section{B. Technique-2: Maximum Likelihood Search for $\varphi$ based on $\varphi_{f}$}

The second technique is based on searching for the most likely value of $\alpha$ depending on the value of $\varphi_{f}$. The search is made on the characteristic curve $g_{v}(\theta \mid \alpha)$ which is generated by estimating the signal to noise ratio $\rho$ for all possible values of $\alpha$ and minimizing the error given by $\epsilon=\left|g_{v}(\theta \mid \alpha, \rho)-\varphi_{f}\right|$. The two possible solutions that minimize $\epsilon$ are then given by

$$
\tilde{\varphi}_{i}=\arg \min _{\alpha}\left\{\left|\varphi_{f}-g_{v}(\theta \mid \alpha, \rho)\right|\right\}
$$

where $i=1,2$. Since we have two possible solutions we perform a simple correlation and match process with the original signal $r$ to select the correct one. The correlation process is given by

$$
\lambda\left(\tilde{\varphi}_{i}\right)=\left|r \exp \left(-j \tilde{\varphi}_{i}\right)\right|
$$

Then, the bias corrected phase based on (20) and (21) is given by

$$
\varphi=\arg \max _{\tilde{\varphi}_{i}}\left\{\lambda\left(\tilde{\varphi}_{i}=\arg \min _{\alpha}\left\{\left|\varphi_{f}-g_{v}(\theta \mid \alpha, \rho)\right|\right\}\right)\right\}
$$

The above method is more computationally complex than the previous one (Technique-1) due to the searching process. The searching process however can be further improved based on various searching techniques which are not treated in this paper. For example, the searching can be vastly improved based on the value of $\varphi_{f}$ itself and also on the estimate of $\rho$ by having the prior knowledge of $g_{v}(\theta \mid \alpha)$ based on $\varsigma=2 N \rho$. The function $\Gamma_{\theta}\left(\varphi_{f}\right)$ in the modified block diagram in Figure 5 represents the operations involved in (22) to compute the bias-corrected phase. Furthermore, the characteristic equation can also be expanded using Taylor series coefficients as polynomials (typically up to the order of seven to match a sine curve between $-\pi$ and $\pi$ ), and the mapping of $\Gamma_{\theta}$ can be obtained by finding the roots of the polynomial which we do not treat in this paper. 


\section{NumericAl EXAMPLES OF LiNEAR PHASE TRACKING}

Here we present some numerical examples considering FIR and IIR filters to explain the bias problem using linear systems and the corresponding bias correction techniques that we presented. The FIR filter considered is a 240-tap low-pass filter generated using the frequency sampling method with cut-offs at normalized frequency points $2 f / f_{s}=[0,0.025,0.026,1]$ with the corresponding frequency domain magnitudes of [1, $1,0,0]$. The IIR filter on the other hand is a low-pass Butterworth filter with six poles given by the transfer function $H(z)=B(z) / A(z)$, where $B(z)=1 e-9 \times\left[0.0141,0.0849^{z-1}, 0.2122 z^{-2}, 0.2829 z^{-3}, 0.2122 z^{-4}\right.$ $\left., 0.0849 z^{-5}, 0.0141 z^{-6}\right]$ and $A(z)=\left[1.0000,-5.8786 z^{-1}, 14.4004 z^{-2},-18.8153 z^{-3}, 13.8294 z^{-4},-5.4216 z^{-5}\right.$ , $\left.0.8857 z^{-6}\right]$. In all of our examples we assume perfect estimation of the signal to noise ratio $\rho$. Figure 6 shows the simulation results of the output phase for the FIR/IIR filters before and after bias correction. Note that the FIR/IIR filters considered here are narrow band filters which are used to reduce the variance to clearly show the mean error (the bias) of the output phase. For phase tracking using the FIR filter, the true phase value was set to $\alpha=36^{\circ}$ with $\varsigma=-10 d B$, and from the figure we see that the bias at the output seems to be quite significant deviating from the true phase value $\alpha$. For the IIR case, the true phase value was set to $\alpha=-42^{\circ}$ with $\varsigma=-5 d B$, and from the simulation results we clearly see the effect of the bias with linear phase tracking. Figure 6 also shows the phase output with bias correction, and when compared to the results with the no bias correction case the improvement is clearly seen. Figure 7 shows the improvement in the mean phase error using the two bias-correction techniques for $\varsigma=-5 d B$. From the figure we see that the linear method for bias correction (Tech-1) fails when $\alpha$ is increased as expected, and the maximum likelihood search method performs well for all values of $\alpha$. Note that the results shown in Figure 7 are for narrow band linear systems to explicitly show the performance of the proposed bias correcting techniques. Figure 8 shows the root mean square error (RMSE) of the phase for the phase estimates with and without bias correction methods together with the Cramer-Rao bound (CRB), where the $\mathrm{CRB}$ is given by $C R B=1 / \varsigma$ [4]. From the figure we see that the scaling operation of the phase estimates with $1 / K_{p}$ for bias correction Tech-1 increases the RMSE of the phase for lower values of $\varsigma$ and reaches the CRB for higher values of $\varsigma$. The RMSE for the phase estimates using Tech- 2 on the other hand reaches the CRB at higher values of $\varsigma$, and approaches the limit of $\pi / \sqrt{3}$ radians for lower values $\varsigma$. It also closely follows the RMSE curve for the case of no bias correction with a slight deviation between $-13 \mathrm{~dB}<\varsigma(\mathrm{dB})<3 \mathrm{~dB}$. 


\section{NONLINEAR PHASE TRACKING}

We present two nonlinear techniques, namely the sequential Monte-Carlo method and the arctan-phasedetector based D-PLL for bias-free phase tracking. The sequential Monte Carlo method for tracking, namely the particle filtering method [35], is usually used for applications with non Gaussian noise, and can be also seen as a mode tracking technique. Here we adopt this technique to track and estimate the phase to eliminate the bias. In [36]-[39], phase tracking using particle filter (Bayesian methods) has been treated considering the phase noise to be Gaussian. In our work, we present phase estimation considering the phase noise model described in (5) using the sequential Monte Carlo method (particle filters) with the aim of achieving bias-free phase estimates. The D-PLL on the other hand is a well known technique for phase tracking and we consider this technique in regards to bias-free phase estimation. In our work, we present a second-order D-PLL and the performance of the loop for bias-free phase tracking. Another estimator that can be considered as a bias-free estimator is the ML-based correlator estimator [30], which maximizes the energy at the output of the correlator bank using a set of local values for $\alpha$ and performing correlation with the received signal $r$ without using the arctan function. We do not treat this technique in this paper but mention it in the interest of the topic covered here.

\section{A. Sequential Monte-Carlo Method for Bias Free Phase Tracking}

By using the discrete signal model and the posteriori density function for the phase, presented in (1) and (5) respectively, we propose a sequential Monte-Carlo based signal processing technique for bias-free phase estimation. The idea behind this technique is to construct the posteriori density function of the phase based on the random samples observed and then compute the estimate based on the constructed density function. The idea of constructing the posteriori density function comes from the theory of Bayesian estimation techniques [1] in signal processing. The sequential Monte Carlo method approaches the optimal Bayesian estimator when the number of samples becomes large [35]. The Bayesian rule for the $n^{\text {th }}$ iterative estimation (where $n$ represents the time index) of the posteriori density $p\left(\alpha_{n} \mid r_{n}\right)$ is given by

$$
p\left(\alpha_{n} \mid r_{n}\right)=\frac{p\left(r_{n} \mid \alpha_{n}\right) p\left(\alpha_{n} \mid r_{n-1}\right)}{p\left(r_{n} \mid r_{n-1}\right)}
$$

where the denominator is computed from

$$
p\left(r_{n} \mid r_{n-1}\right)=\int p\left(r_{n} \mid \alpha_{n}\right) p\left(\alpha_{n} \mid r_{n-1}\right) d \alpha_{n}
$$

Then, by using the MMSE criterion, the Bayesian estimate is given by

$$
\hat{\alpha}=\int \alpha_{n} p\left(\alpha_{n} \mid r_{n}\right) d \alpha_{n}
$$


The estimator in (25) can give rise to the bias problem again due to the estimation of the mean from the posteriori density function which is bi-modal as we described in the previous sections. Therefore, we also consider the MAP criterion to estimate the phase given by

$$
\hat{\alpha}=\arg \max _{\alpha_{n}}\left\{p\left(\alpha_{n} \mid r_{n}\right)\right\}
$$

Having formulated the estimators as in (25) and (26) based on the Bayesian principles, now we present the particle filtering technique [35] to construct the posteriori density function of the phase in order to perform the estimates. Note that we consider $N=1$ here (where $N$ is defined in equation (4)), and from this point onwards we consider the same unless explicitly specified. We also consider the signal model in (1) with an unknown phase $\alpha_{n}=\alpha$ considered to be a constant in the time interval of our interest (i.e. within the time period for a Bayesian estimate given by (25) or (26)), and which (i.e. the phase) can evolve over the length of the transmitted sequence outside this time period. Then, based on the observations $\theta_{n}=g\left(\alpha+\theta_{v}\right)$, where $\theta_{n}$ is given by (7) at time instance $n$, we generate the posteriori density function of $\alpha$ characterized by the pair $\left\{w_{k}^{n}, \alpha_{k}^{n}\right\}$, which is given by

$$
p\left(\alpha \mid \theta_{n}\right) \approx f_{\alpha}\left(w_{k}^{n}, \alpha_{k}^{n}\right)=\sum_{k=1}^{M} w_{k}^{n} \delta\left(\alpha-\alpha_{k}^{n}\right) \quad \text { with } \sum_{k=1}^{M} w_{k}^{n}=1
$$

where $\alpha_{k}^{n}$ are known as the particles drawn from the posteriori density function (known as the optimal importance function), $w_{k}^{n}$ are the corresponding weights assigned to the particles for the $n^{\text {th }}$ time sample imitating the true posteriori density function of the phase, and $M$ is the total number of particles considered. The weights are assigned with initial values of $w_{k}^{0}=1 / M$ at $n=0$, and are iteratively computed using the optimal importance function. Note that the posteriori density function $p\left(\alpha \mid \theta_{n}\right)$ is the same as the density function described in equation (5). From [35], for the optimal importance function, the weights are iteratively computed by

$$
\begin{gathered}
\bar{w}_{k}^{n+1}=\bar{w}_{k}^{n} p\left(\theta_{n+1} \mid \alpha\right) \\
w_{k}^{n+1}=\bar{w}_{k}^{n} / \sum_{k=1}^{M} \bar{w}_{k}^{n}
\end{gathered}
$$

Since $\alpha$ is an unknown constant the function $p\left(\theta_{n+1} \mid \alpha\right)$ becomes proportional to the density function described in (5) given by $f_{\theta}\left(\alpha_{k}^{n} \mid \rho, \theta_{n+1}\right)$. Then by ignoring the constant terms in $f_{\theta}\left(\alpha_{k}^{n} \mid \rho, \theta_{n+1}\right)$ we can update the weights as $\bar{w}_{k}^{n+1}=\bar{w}_{k}^{n} \Lambda$, where

$$
\Lambda=\Upsilon \exp \left(\Upsilon^{2}\right)(1-Q(\Upsilon)) \text { with, } \Upsilon=\cos \left(\alpha_{k}^{n}-\theta_{n+1}\right)
$$

In (30), the term involving the $Q($.$) function may be ignored when the necessary conditions are satisfied$ 
as per the expression in (5), hence (30) may reduce to $\Lambda \approx\left|\Upsilon \exp \left(\Upsilon^{2}\right)\right|$. The newly computed particle weights are then used to estimate the phase by taking the maximum of the discrete weights $w_{k}^{n}$, given by

$$
\hat{\alpha}=\arg \max _{\alpha_{k}^{n}}\left\{f_{\alpha}\left(w_{k}^{n}, \alpha_{k}^{n}\right)\right\}
$$

After a few iterations (i.e. few estimations), some of the particles will have almost zero weights which are no longer required for the estimation process known as the degeneration affect of the computed density function. In such situations resampling techniques are used to reduce the degeneration affect associated with the estimation process by discarding the particles with negligible weights. In Section-VIII we present simulation results to show the performance of the particle filtering approach for phase estimation discussed in this section.

\section{B. Digital Phase-Locked Loop for Bias Free Phase Tracking}

The second nonlinear technique that we consider for bias-free phase tracking is the Digital Phase-locked loop (D-PLL) using the arctan function as the phase detector in the complex envelope form [26]. Such a loop model is generally known as the tan-lock loop in the literature [15]-[18]. Here we present the arctan-based D-PLL in the context of the topic treated in this paper which is bias-free phase tracking, $\boldsymbol{a}$ topic which has not been touched upon in the literature of PLL.

1) Loop Design: A typical second-order D-PLL has a phase detector (PD), a loop filter $\mathrm{D}(\mathrm{z})$ and a Number Controlled Oscillator (NCO) V(z) as depicted in Figure 9. The double lines in the figure represent complex signals and the single lines represent real signals. The equivalent mathematical model of the PD is represented in Figure 9 showing the nonlinearity associated with the arctan phase detector by expressing it with its characteristic function as described in equations (8) and (9). The output of the NCO is given by $x[n]=\exp \{-j \theta[n]\}$ which is multiplied by $r[n]$ using a complex multiplier to generate $e[n]$. The output of the $\arctan$ function (represented by the $g_{\theta}($.$) function) is then given by \varphi_{e}[n]=\arg \{e[n]\}=g_{\theta}\left(\phi[n]+\theta_{v}\right)$ where $\theta_{v}$ is the same zero mean noise process defined in (7), and $\phi[n]=\alpha-\theta[n]$ is the true phase error between the estimated phase and the input phase. From $\varphi_{e}[n]$, in the noiseless case, we see that the phase detector output becomes the true phase error, that is $\varphi_{e}=\phi$. Considering the noiseless case the loop can be modeled as a linear system given by the closed loop transfer function

$$
H(z)=\frac{D(z) V(z)}{1+D(z) V(z)}
$$

where $V(z)=k_{v} /[z-1]$ and $D(z)=a_{f} z /\left[z-\left(1-a_{f}\right)\right]$. Furthermore in the noiseless case, given the input signal $r$ as in (1), the above second-order loop will drive the phase error $\phi[n]$ (and hence $\varphi_{e}[n]$ ) to 
a steady state value of $\varphi_{e}^{s}=0$, which can be proved by using the final value theorem as,

$$
\varphi_{e}^{s} \triangleq \lim _{n \rightarrow \infty} \varphi_{e}[n]=\lim _{z \rightarrow 1} \frac{(z-1)}{z} \varphi_{e}(z)
$$

where $\varphi_{e}(z)$ is the z-transform of $\varphi_{e}[n]$. By expressing $\varphi_{e}(z)$ as $\varphi_{e}(z)=\Theta_{i n}(z)[1-H(z)]$, where $\Theta_{i n}(z)$ is the z-transform of the input phase $\alpha$ given by $\alpha z /(z-1)$, the steady state phase error $\varphi_{e}^{s}$ becomes

$$
\varphi_{e}^{s}=\lim _{z \rightarrow 1} \frac{(z-1)}{z} \frac{\alpha z}{(z-1)}[1-H(z)]=0
$$

With no further disturbances at the input, the phase error will remain at zero, known as the steady state mode, and hence giving us $\theta[n]=\alpha$. This itself is a proof of bias-free phase tracking in the noise free case. In the noisy case however, when there is additive disturbances at the input, the steady state phase error will experience a jitter around the mean phase error value which is zero, and hence the steady state phase error process can be treated as a zero mean process. We note that, having a zero mean phase error process is the key feature here to attain bias-free phase tracking which we explain in detail in the next section. The steady state phase error distribution can be found using the Chapman-Kolmogorov equation by modeling the phase error as a second-order Markov process [31],[14],[20] which we do not treat in this paper, instead we consider the open-loop steady state distribution which is easier to analyze and serves our purpose to illustrate the bias-free phase tracking process in the presented D-PLL. In the next section we present the bias-free phase tracking performance of the arctan based D-PLL presented in this section.

\section{ViII. Performance Comparison of Bias-Free Phase Tracking}

The relative performance of the proposed linear and nonlinear techniques for bias-free phase tracking is presented here. Figure 10 depicts the mean phase estimation/tracking performance of the proposed techniques which we further explain subsequently.

\section{A. Performance of the Sequential Monte Carlo Method}

The expected value of the phase estimates from the MAP-based sequential Monte-Carlo estimates is depicted in Figure 10 for $\rho=-7 \mathrm{~dB}$, with $\mathrm{M}=100$ and $N=1$. From the figure we clearly see that there exist no bias and the mean phase estimates match very closely to the true phase values $\alpha$ even for higher values of $\alpha$. Even though the particle filter based method provides good bias-free phase tracking performance the complexity associated with it however in computing the particle weights and the corresponding phase estimates is a major drawback in designing such a technique. Furthermore, an important point to be 
mentioned here is that if $\alpha_{n}$ does not evolve with time but remains static then particle filter algorithm can fail, and the treatment of such is beyond the scope of this paper.

\section{B. Performance of arctan phase detector based D-PLL}

The performance of the D-PLL for bias-free phase tracking is presented considering 1) the open-loop steady state distribution and 2) the steady state mean phase output.

1) Open-Loop Steady State Distribution: As we see from the block diagram of the D-PLL, the loop during the acquisition mode feeds back the new estimates of the phase $\theta$ and brings the phase error $\phi$ to zero, and hence in the steady state the distributions of $\phi$ and $\varphi_{e}$ become zero mean. In other words, D-PLL brings back the phase error distribution to a zero mean distribution due to the negative feedback process. Considering the phase detector model of the D-PLL (Figure 9) and by using the density function in (5) the open-loop density of $\varphi_{e}$ can be expressed as $f_{\varphi_{e}}\left(\varphi_{e}\right)=f_{\theta}\left(\varphi_{e} \mid \rho, 0\right)$ with $\mathrm{N}=1$ [26]. Likewise, the open-loop density for the phase error process $\phi$ is approximated as $f_{\phi}(\phi) \simeq f_{\theta}\left(\phi \mid \frac{\rho \mathrm{B}_{i}}{2 \mathrm{~B}_{\mathrm{L}}}, 0\right)$ for small values of $B_{L}$ [26], where $B_{i}$ is the input noise bandwidth and $B_{L}$ is the loop bandwidth of the D-PLL defined by $2 \mathrm{~B}_{\mathrm{L}}=\frac{\mathrm{B}_{\mathrm{i}}}{2 \pi j} \oint H(z) H\left(z^{-1}\right) d z$ [20]. Figure 11 shows the open-loop steady state phase distribution together with the simulation results, and from the results we observe how the loop pulls in the phase and centers the density of $\phi$ at zero.

2) Steady State Mean Phase Error: Simulations are performed to analyze the the steady state mean phase error of the D-PLL. Figure 10 shows the mean phase estimates for various values of $\alpha$ with $\varsigma=-7 \mathrm{~dB}$. For comparison, we also present the performance of the arctan-based ML estimates described by (4) and the bias-correction techniques proposed in Section V for linear phase tracking in the same figure. From the figure we clearly observe that there is no bias present in the arctan phase-detector-based D-PLL phase output. We further observe that Technique-2 for bias correction in LS matches the performance of the particle filter and D-PLL based techniques but requires the estimation of SNR.

\section{Numerical Complexity}

The complexity of the proposed techniques with respect to the number of operations required per estimate is presented in Table 1. In the table, $\mathrm{N}$ is the number of samples per ML phase estimate as in (4), $\mathrm{N}_{\mathrm{arg}}$ is the number of operations to calculate the arctan value of a complex vector, $\mathrm{N}_{\varsigma}$ is the number of operations to estimate the $\operatorname{SNR}(\varsigma), \mathbf{M}_{\beta}$ is the number of sample points for $\beta$ representing $g_{v}(\theta \mid \beta)$ as in (9), $\mathbf{M}$ is the number of particles in the particle filter, and $\mathrm{K}$ is the number of iterations to represent the summation in (9) and (11). Based on the expressions for the complexity in the table we observe that the D-PLL outperforms 
the rest of the techniques, whilst noting that it also provides excellent bias performance as observed in Figure 10. The D-PLL however can suffer from acquisition failures and cycle slips [14] which are not treated in this paper.

\section{CONCLUSION}

In this paper we discussed the bias problem associated with the arctan-based ML phase estimator for phase tracking. The bias was identified to be large for lower values of signal to noise ratio and higher values of the phase when linear systems are used for tracking, and our analyses were verified by simulation results. We provided solutions to correct the bias 1) using the phase estimator gain and 2) by using a maximum likelihood search on the characteristics equation of the estimator. Both the techniques need to estimate the SNR of the signal to estimate and correct the bias. The former method performs well for smaller values of phase due to the small angle approximation associated with it, and the latter method performs well for all values of the phase with additional computational complexity. We importantly point out here that the variance of the phase estimates can be reduced by narrowing the bandwidth of the linear tracking system whereas the mean error (bias) can only be reduced by bias correcting techniques such as the ones presented in this paper. Furthermore, two nonlinear techniques were also presented based on sequential Monte-Carlo technique and arctan-based digital phase locked loop technique for unbiased (bias-free) phase estimation, especially at low signal to noise ratio. Results show that the sequential Monte-Carlo tracking based on Bayesian principle shows no bias in the phase estimates at the expense of additional computational complexity. On the other hand, the digital phase locked loop shows excellent phase tracking performance with no bias especially with low computational complexity. Compared to the nonlinear systems for biasfree phase tracking the linear systems require to estimate the signal to noise ratio to correct for the bias. The digital phase locked loop in particular stands out amongst the presented techniques for bias correction considering all the advantages associated with it.

\section{APPENDiX 1}

The following integral is evaluated in this section (derived from [23]).

$$
g_{v}(\theta)=\int_{-\pi}^{\pi} g\left(\beta+\theta_{v}\right) f_{\theta_{v}}\left(\theta_{v}\right) d \theta_{v}
$$

where $\theta_{v}$ is a zero mean random process given by $f_{\theta_{v}}\left(\theta_{v}\right)=f_{\theta}(\theta \mid \rho, \alpha=0)$. Considering the noise free characteristics is a unit ramp function between $-\pi$ and $\pi$ and expanding $g\left(\beta+\theta_{v}\right)$ in its Fourier series 
coefficients (35) can be written as,

$$
g_{v}(\theta)=\sum_{k=1}^{\infty} \int_{-\pi}^{\pi} \frac{(-1)^{k+1}}{k} \sin \left(k\left(\beta+\theta_{v}\right)\right) f_{\theta_{v}}\left(\theta_{v}\right) d \theta_{v}
$$

using trigonometric expansions and identifying the $\int_{-\pi}^{\pi} \sin \left(k \theta_{v}\right) f_{\theta_{v}}\left(\theta_{v}\right) d \theta_{v}=0$ (since $\theta_{v}$ is zero mean), (36) becomes,

$$
g_{v}(\theta)=\sum_{k=1}^{\infty} \int_{-\pi}^{\pi} \frac{(-1)^{k+1}}{k} \sin (k \beta) \cos \left(k \theta_{v}\right) f_{\theta_{v}}\left(\theta_{v}\right) d \theta_{v}
$$

A closed form solution to the integral expression $\int_{\pi}^{\pi} \cos \left(k \theta_{v}\right) f_{\theta_{v}}\left(\theta_{v}\right) d \theta_{v}$ is obtained in [24] as

$$
\int_{\pi}^{\pi} \cos \left(k \theta_{v}\right) f_{\theta_{v}}\left(\theta_{v}\right) d \theta_{v}=0.5 \sqrt{\pi} \exp \left(\frac{-\varsigma}{2}\right)\left[I_{\frac{k-1}{2}}(\varsigma)+I_{\frac{k+1}{2}}(\varsigma)\right]
$$

Therefore, the solution to (40) becomes,

$$
g_{v}(\theta)=\sum_{k=1}^{\infty} \frac{(-1)^{k+1}}{k} \sqrt{\pi \varsigma} \exp \left(-\frac{\varsigma}{2}\right)\left[I_{\frac{k-1}{2}}(\varsigma)+I_{\frac{k+1}{2}}(\varsigma)\right] \sin (k \beta)
$$

where $I_{x}($.$) is the modified Bessel function of order x$.

\section{APPENDIX 2}

The expression for the non-central second moment $E\left[\theta^{2}\right]$ of the phase estimates $\theta$ is derived using similar steps in Appendix 1, which is given by

$$
E\left[\theta^{2}\right]=\int_{-\pi}^{\pi} g^{2}\left(\beta+\theta_{v}\right) f_{\theta_{v}}\left(\theta_{v}\right) d\left(\theta_{v}\right)
$$

considering $\theta_{v}$ is a zero mean random process given by $f_{\theta_{v}}\left(\theta_{v}\right)=f_{\theta}(\theta \mid \rho, \alpha=0)$, and that the noise free characteristics is a unit ramp function between $-\pi$ and $\pi$. we can expand $g^{2}\left(\beta+\theta_{v}\right)$ in its Fourier series coefficients, and (40) can be written as,

$$
E\left[\theta^{2}\right]=\sum_{k=1}^{\infty} \int_{-\pi}^{\pi} \frac{(-1)^{k}}{k^{2}} \sin \left(k\left(\beta+\theta_{v}\right)\right) f_{\theta_{v}}\left(\theta_{v}\right) d \theta_{v}
$$

Again, using trigonometric expansions and following the steps in Appendix 1, (41) becomes,

$$
E\left[\theta^{2}\right]=\frac{\pi^{2}}{3}+2 \sqrt{\pi \varsigma} \exp \left(-\frac{\varsigma}{2}\right) \sum_{k=1}^{\infty} \frac{(-1)^{k}}{k^{2}}\left[I_{\frac{k-1}{2}}\left(\frac{\varsigma}{2}\right)+I_{\frac{k+1}{2}}\left(\frac{\varsigma}{2}\right)\right] \cos (k \beta)
$$

Note that, a similar expression is also presented in [23] for $\beta=0$ only, that is for the variance of $\theta_{v}$, but the expression that we derived here in (42) is for all values of $\beta$ between $-\pi$ and $\pi$. 


\section{REFERENCES}

[1] S. M. Kay, Fundamentals of Statistical Processing, Volume 1: Estimation Theory, Prentice Hall, New Jersey 1993.

[2] K. Peters and S. Kay, "Unbiased estimation of the phase of a sinusoid," IEEE International Conference on Acoustics, Speech, and Signal Processing Vol.2, 17-21 May, 2004.

[3] J. S. Lee and J. H. Hughen, ”An Optimum Phase Syncronizer in a Partially Coherent Receiver," IEEE Trans on Aerospace and Electronic Systems, Vol.AES7, No.4, pp 652-661, July 1970.

[4] D.C. Rife and R. Boorstyn, "Single-tone parameter estimation from discrete-time observations," IEEE Transactions on Information Theory, Vol. IT-20, No.5, pp 591-598, Sep 1974.

[5] R. Reggiannini, "A Fundamental Lower Bound to the Performance of Phase Estimators Over Rician-Fading Channels," IEEE Transactions on Communications, Vol.45, No.7, pp 775-778, July 1997.

[6] A.J. Viterbi and A.M. Viterbi, ”Nonlinear estimation of PSK-modulated carrier phase with application to burst digital transmission," IEEE Transactions on Information Theory, Vol.IT-29. No.4, pp 543-551, July 1983.

[7] B. E. Paden, "A matched nonlinearity for phase estimation of a PSK-modulated carrier", IEEE Transactions on Information Theory, Vol.32 No.3, pp 419-422, May 1986.

[8] P. Kam, 'Maximum Likelihood Carrier Phase Recovery for Linear Suppressed-Carrier Digital Data Modulations', IEEE Transactions on Communications, Vol.34, No.6, pp 522-527, June 1986.

[9] R. Di Girolamo and T. Le-Ngoc, "Frequency Independent Nonlinear Feedforward Phase Estimator," Wireless Personal Communications Journal, Kluwer Academic Publishers, Vol.5, No.1, pp 19-50, July 1997.

[10] Y. Surrel, "Additive Noise Effect in Digital Phase Detection," Journal on Applied optics, Optical Society of America, Vol.36, No.1, pp 271-276, Jan 1997.

[11] H. Fu and P. Kam, "MAP/ML Estimation of the Frequency and Phase of a Single Sinusoid in Noise", IEEE Transactions on Signal Processing, Vol.55, No.3, pp 834-845, March 2007.

[12] B. C. Lovell and R. C. Williamson, "The Statistical Performance of Some Instantaneous Frequency Estimators," IEEE Transaction on Signal Processing, Vol.40, No.7, pp 1708-1723, July 1992.

[13] V. Clarkson, P. J. Kootsookos, and B. G. Quinn, ”Analysis of the Variance Threshold of Kays Weighted Linear Predictor Frequency Estimator," IEEE Transactions on Signal Processing, Vol.42, No.9, pp 2370-2379, Sep 1994.

[14] F.M. Gardner, Phaselock Techniques, Third Edition, New York: John Wiley and Sons, 2005.

[15] J. C. Lee and C. K. Un, "Performance Analysis of Digital Tanlock Loop," IEEE Transactions on Communications Vol.30, No.10, pp 2398-2411, Oct 1982.

[16] C. A. Pomalaza-Raez, "Noise Analysis of a Digital Tanlock Loop," IEEE Trans on Aerospace Electronic Systems, Vol.24, Iss.6, pp 713-718, Nov 1988.

[17] A. H. Makarious and P. G. Farrell, ”Novel p.s.k. tanlock loop," IEEE Elect. Letters, Vol.16, Iss.25, pp 957 - 958, Dec 1980.

[18] W. D. Cho and C. K. Un, ”On improving the performance of a digital tanlock loop,” Proceedings of the IEEE, Vol.75, Iss.4, pp 520 - 522, Apr 1987.

[19] A. Weinberg and Bede Liu, ”Discrete Time Analyses of Nonuniform Sampling First- and Second-Order Digital Phase Lock Loops," IEEE Transactions on Communications, Vol. 22, No.2, pp 123-137, Feb 1974.

[20] W. Lindsey and C. Chie, ”A Survey of digital phase-locked loops," Proc. of the IEEE, Vol.69, Iss.4, pp 410-431, Apr 1981.

[21] E. H. Shetelmen, "The Transfer Characteristic of a Linear Phase Detector when Its Input Signal-Noise Ratio Is Small," IEEE proceedings, Vol.55, Iss.5, pp 694-694 May 1967. 
[22] B.N. Biswas, et-al. "Phase Detector Response To Noisy and Noisy Fading Signals," IEEE Trans AeroSpace and Electronic Sys Vol.AES-16, No.2, pp 150-158, Mar 1980.

[23] Werner Rosenkranz, "Phase-Locked Loops with Limiter Phase Detectors in the Presence of Noise," IEEE Transactions on Communications, Vol.30, No.10, pp 2297-2304, Oct 1982.

[24] J.C. Springet and M. K. Simon, "An Analysis of the Phase Coherent-Incoherent Output of the Bandpass Limiter," IEEE Transactions on Communications Technology, Vol.19, No.1, pp 42-49, Feb 1971.

[25] S. Kandeepan and S. Reisenfeld, "Phase detector models and their performances for IF/baseband frequency recovery for complex envelope based DSP implemented PLL," IEEE ICCS'02, 25-28 Nov, 2002

[26] S. Kandeepan and S. Reisenfeld, "Frequency tracking and acquisition with a four-quadrant arctan phase detector based digital phase locked loop," IEEE ICICS, 15-18 Dec. 2003,

[27] S.Kandeepan, B. Rohani and M. Caldera, "Performance Evaluation of a Linear Predictor Frequency Estimator for Mobile Flat Fading Wireless Channels," to appear on IEEE Transactions of Vehicular Technology.

[28] S Kandeepan, Synchronisation techniques for digital modems, PhD thesis, University of Technology, Sydney, 2003

[29] U. Mengali and A. N. D’Andrea, Syncrhonization Technqiques for Digital Receivers (Applications of Communicatiosn Theory), Plenum Press, 1997.

[30] H. Meyer and G. Ascheid, Synchronisation in Digital Communications, vol-1, John Willey and Sons, 1990.

[31] A. J. Viterbi, Principles of Coherent Communications, McGraw-Hill, 1966.

[32] W. C. Lindsey and M. K. Simon, Telecommunication Systems Engineering, Englewood Cliffs, NJ Prentice Hall, 1973.

[33] S. C. Gupta, ”Phase-Locked Loops," Proceedings of IEEE vol. 63, pp 291-306, Feb 1975.

[34] B. O. Anderson and J. B. Moore, Optimal Filtering, Dover Books on Engineering, 2005.

[35] M. S. Arulampalam, S. Maskell, N. Gordon, and T. Clapp, "A Tutorial on Particle Filters for Online Nonlinear/Non-Gaussian Bayesian Tracking," IEEE Transactions on Signal Processing, Vol.50, No.2, pp 174-188, Feb 2002.

[36] J. Dauwels and H. A. Loeliger, "Phase Estimation by Message Passing," IEEE ICC Proceedings, 20-24 June 2004, Paris

[37] J. J. Martinez-Espla, et.al, ”A Particle Filter Approach for InSAR Phase Filtering and Unwrapping," IEEE Trans On Geoscience And Remote Sensing, Vol.47, No.4, pp 1197-1211, Apr 2009.

[38] P.O Amblard, J. M. Brossier and E. Moisan, ”Phase Tracking: What do we gain from optimality? Particle Filtering versus Phase-Locked Loops, ” Elsevier Journal on Signal Processing, Vol.83, Iss.1, pp 151-167, Jan 2003.

[39] D. Le Ruyet, T. Bertozzi and N. Paul, "Particle Filtering for Iterative Data And Phase Estimation," IEEE ICASSP 2006 Proceedings, Vol.4, 14-19 May 2006, Toulouse.

[40] G. Colavolpe, A. Barbieri and G. Caire, "Algorithms for Iterative Decoding in the Presence of Strong Phase Noise," IEEE journal on selected areas in communications (JSAC), Vol.23, Iss.9, pp 1748-1757, Sep 2005.

[41] A. N. DAndrea, U. Mengali, and G. M. Vitetta, ”Approximate ML decoding of coded PSK with no explicit carrier phase reference," IEEE Transactions on Communications, Vol.42, Iss.234, pp 1033-1040, Feb./Mar./April 1994.

[42] G. Colavolpe and R. Raheli, ”Noncoherent sequence detection,” IEEE Trans on Comm., Vol47, Iss9, pp1376-1385, Sep 1999.

[43] N. M. Blachman, "Gaussian Noise-Part II: Distribution of Phase Change of Narrow-Band Noise Plus Sinusoid," IEEE Transactions On Information Theory, Vol.34, No.6, pp 1401-1405, Nov 1988.

[44] R. F. Pawula, S. O. Rice and J. H. Roberts, ”Distribution of the Phase Angle Between Two Vectors Pertubed by Gaussian Noise," IEEE Transactions on Communications, Vol.30. No.8, , pp 1828-1841, Aug 1980.

[45] W. R. Bennett, ”Methods of solving noise problems," Proceedings of the IRE, Vol.44, No.5, pp 609-638, May 1956. 
[46] S. Kandeepan, S. Reisenfeld, Performance Analysis of a Logarithmic Based Phase Detector for Tan-Locked Loops, IEEE conference on Personal Indoor Mobile and Radio Communications (PIMRC), Athens, 2007

[47] S. Kandeepan, Omar. H, Qian, A Complex Signal Based Digital Phase-Locked Loop with arctan Phase Detector Implemented on High Speed FPGA, IEEE conf on Info Comm \& Signal Processing 2007, Singapore

[48] S. Kandeepan, A Software Phase Locked Loop from Theory to Practice: TMS320C6000 DSP Based Implementation and Analysis, AusWireless conf 2006, Sydney March 13-16.

[49] S. Kandeepan, S. Reisenfeld. Frequency Error Correction for OFDM based Multicarrier Systems and Performance Analysis, IEEE Int Conf on Information, Communications and Signal Processing ICICS, 6-9 Dec, Bangkok, 2005.

[50] S. Kandeepan, S. Reisenfeld, Performance Analysis of a Digital Phase-Locked Loop with a Hyperbolic Nonlinearity, IEEE International Conference on Information, Communications and Signal Processing, 6-9 Dec, Bangkok, 2005.

[51] S. Kandeepan, and S. Reisenfeld, Acquisition Performance of a Digital Phase Locked Loop with a Four-Quadrant arcane based Phase Detector, IEEE International Symposium on Intelligent Signal Processing and Communications, Nov 2004.

[52] S. Kandeepan, and S. Reisenfeld, Fast Doppler Tracking DSP-Based Earth Station Modem for LEO Satellite Applications, IEEE Int Symp on Intelligent Signal Processing and Communications, Nov 2004

[53] S. Kandeepan, and S. Reisenfeld, Performance Analysis of DPLL with statistical filters for carrier synchronisation, IEEE International Conference on Signal Processing and Communications, Dec 2004.

[54] S. Kandeepan and S. Reisenfeld, Performance Analysis of a Correlator Based Maximum Likelihood Frequency Estimator, IEEE International Conference on Signal Processing and Communications, Dec 2004

[55] S. Kandeepan and S. Reisenfeld, "Frequency Tracking and Acquisition with a Four-Quadrant arctan Phase Detector Based Digital Phase-Locked Loop” Fourth International Conference on Information Communications \& Signal Processing and the IEEE Pacific-Rim Conference on Multimedia Proceedings, Dec 16-18 2003, Singapore.

[56] S. Kandeepan. and S. Reisenfeld, "DSP Based Frequency Estimation Techniques and Their Relative Performances", Symp on Communication Systems, Networks and Digital Signal Processing (CSNDSP2002), July 15-17, Staffordshire.

[57] S. Kandeepan and S. Reisenfeld, "Frequency Jitter of a Digital Phase-Locked Loop and comparison with a Modified CRB", IEEE International Conference on Communication Systems 2002, Nov 25-29. Singapore.

[58] S. Kandeepan and S. Reisenfeld, "A Complex Envelope Based 2nd Order Digital Phase Locked Loop and its Performance”, IEEE International Symposium on Intelligent Signal Processing and Communication Systems 2001(ISPACS 2001) Proceedings of, Nov 20-23, Nashville 


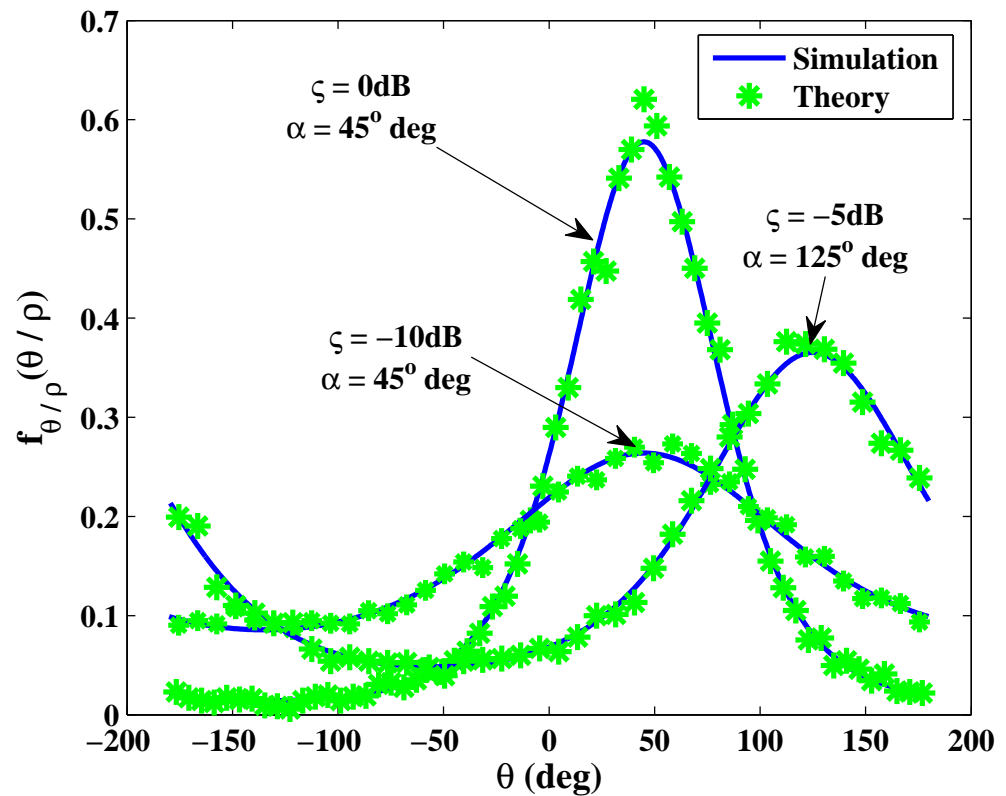

Fig. 1. density function of the phase estimates, comparing the theoretical and the simulation results.

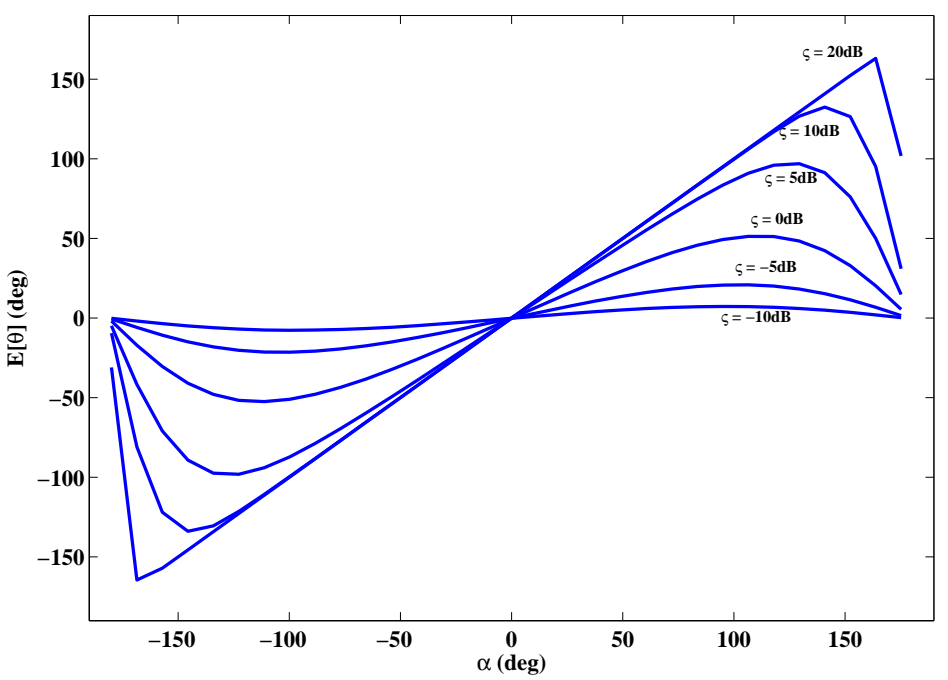

Fig. 2. Characteristics curves of the arctan ML phase estimator 


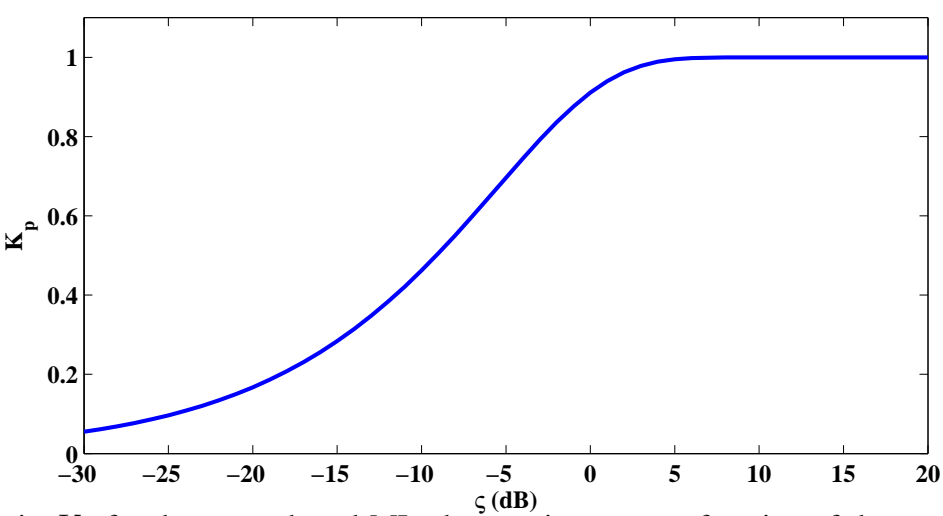

Fig. 3. Phase estimator gain $K_{p}$ for the arctan-based ML phase estimator as a function of the $\varsigma$

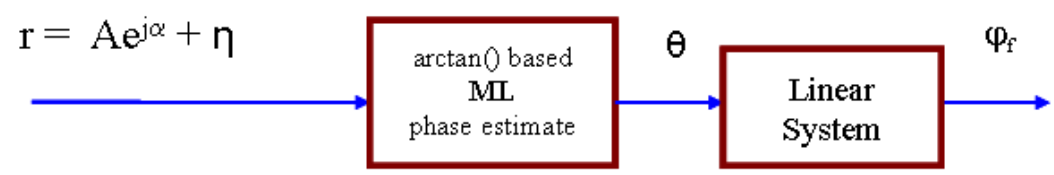

Fig. 4. Phase tracking using linear systems based on ML phase estimates

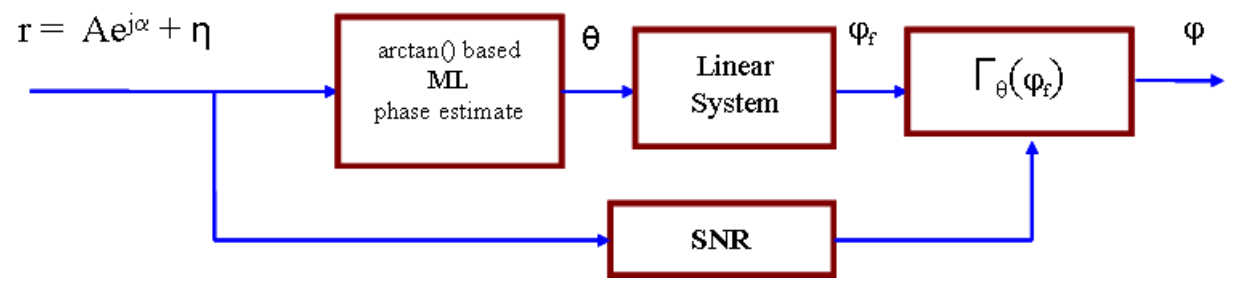

Fig. 5. Modified block diagram for for phase tracking with bias correction

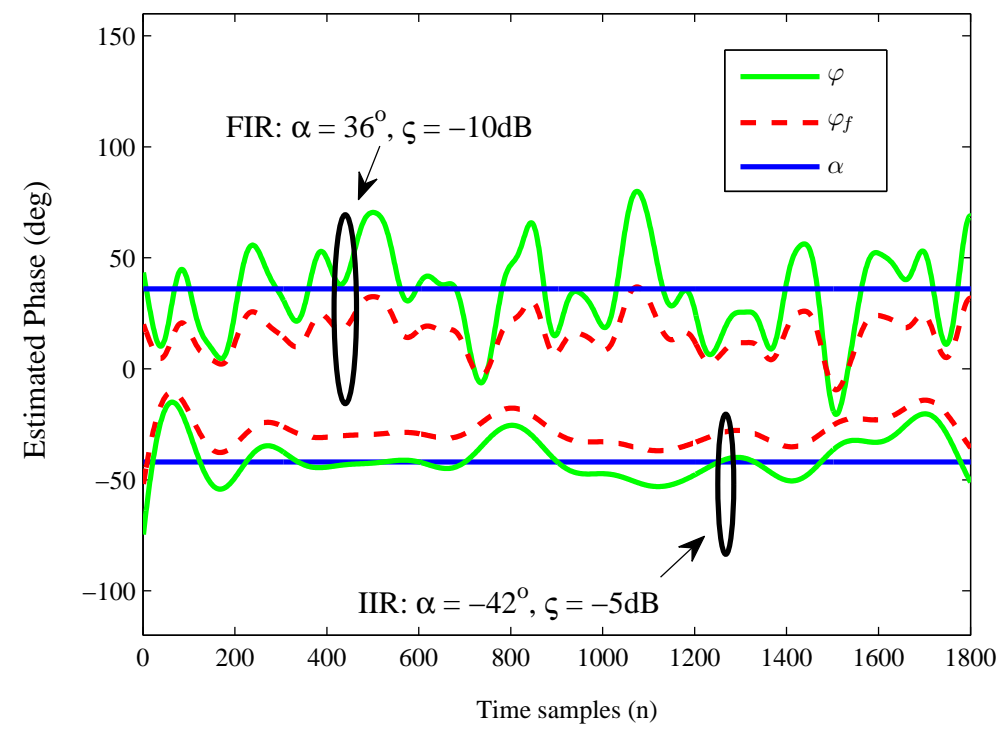

Fig. 6. Narrow band linear system's response to ML phase estimates with $(\varphi)$ and without $\left(\varphi_{f}\right)$ bias correction for $K_{0}=1$, case1-FIR: $\alpha=36^{\circ}, \varsigma=-10 d B$, case2-IIR: $\alpha=-42^{\circ}, \varsigma=-5 d B$ 


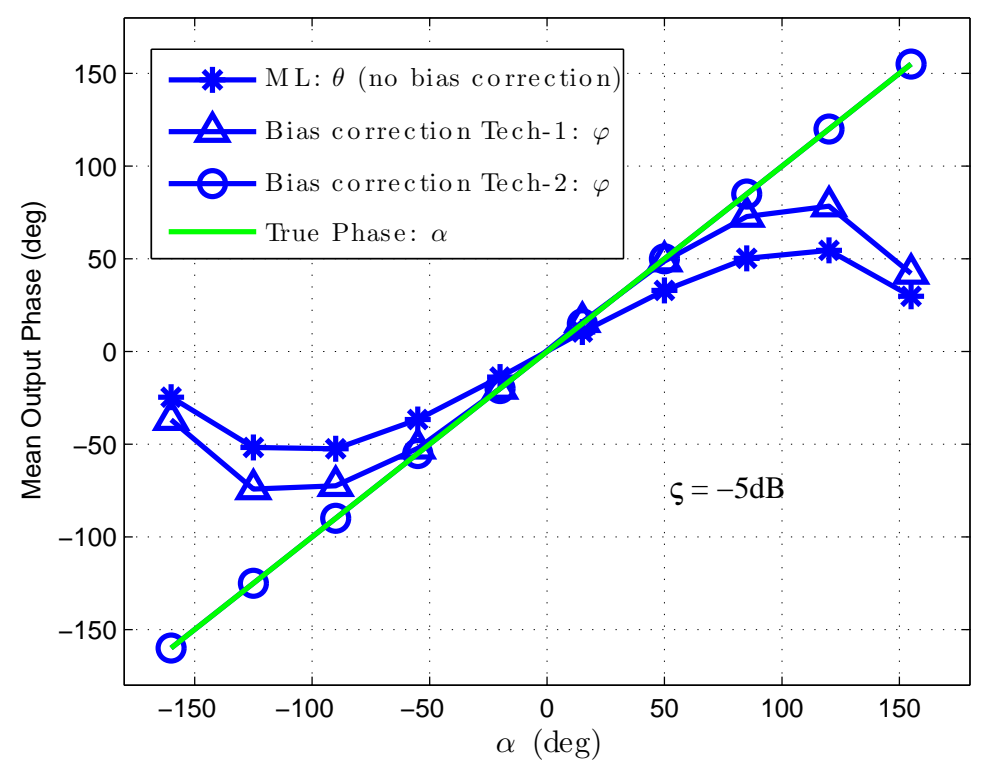

Fig. 7. Mean phase estimates, before and after bias correction for narrow band linear systems

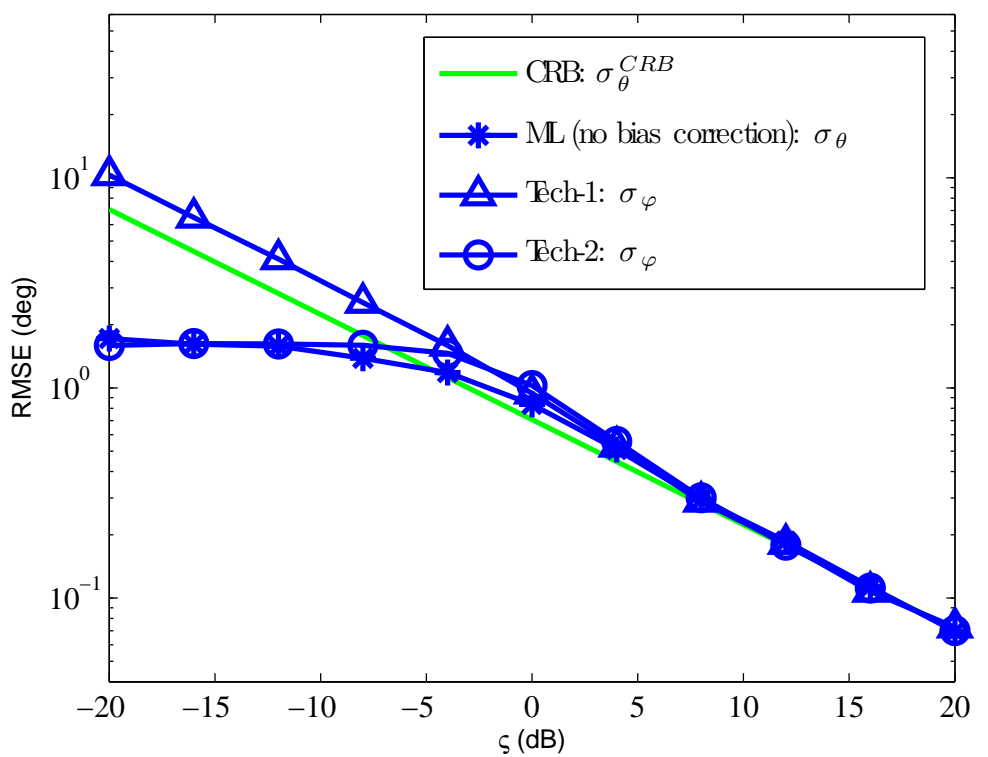

Fig. 8. Root mean square error (RMSE) performances of the phase estimates before and after the bias correction: To study the RMSE, linear systems with the same bandwidth as the noise bandwidth were used

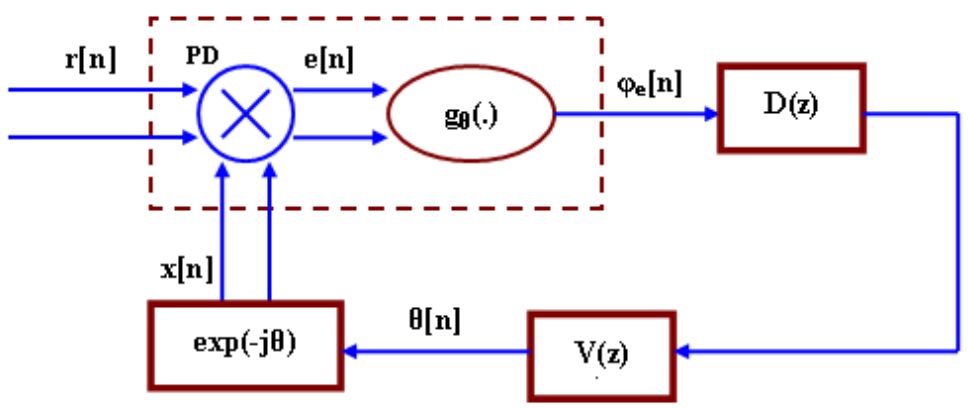

Fig. 9. Block diagram of the D-PLL based for bias-free phase tracking 


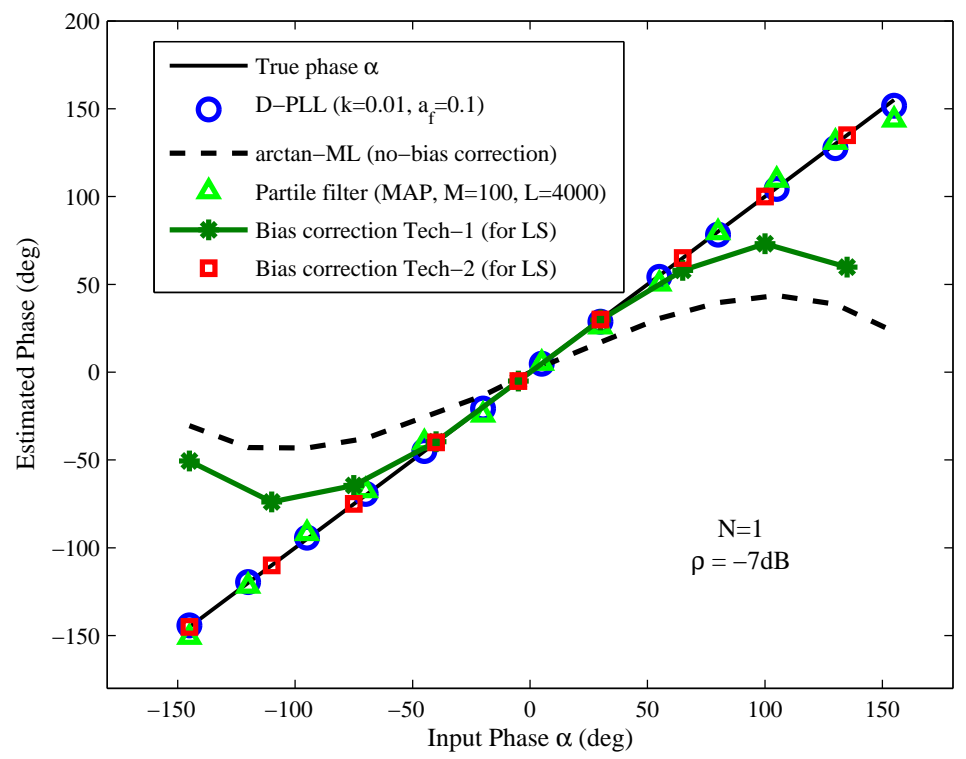

Fig. 10. Comparing the mean phase estimates (bias) using the proposed techniques

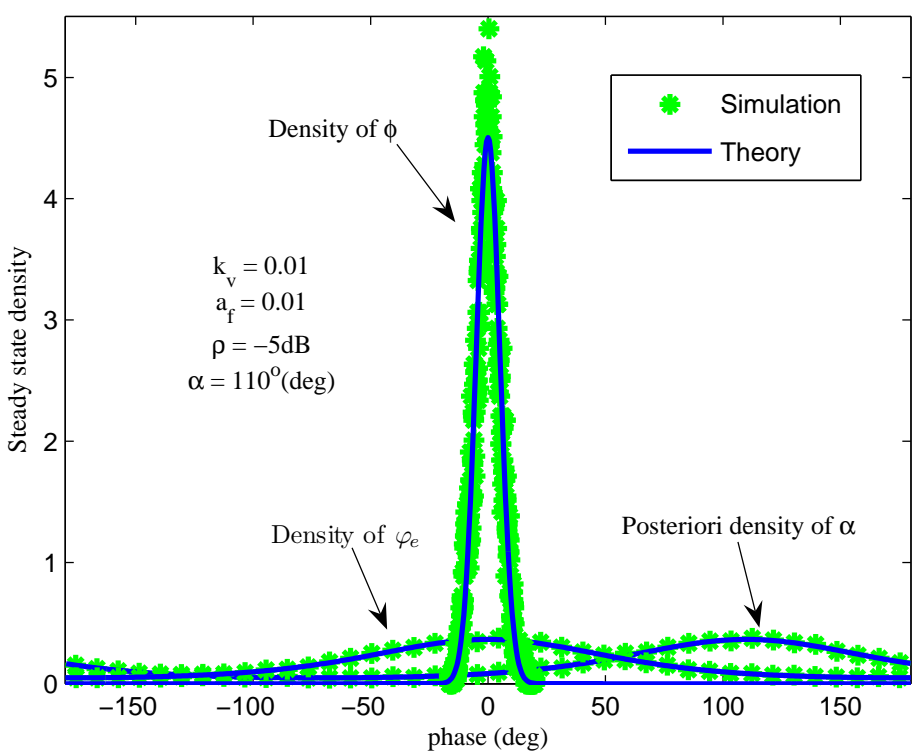

Fig. 11. Steady state (open loop) density functions of the phase processes in the second-order arctan phase detector based D-PLL; $\mathrm{B}_{\mathrm{L}} / \mathrm{B}_{\mathrm{i}}=2.5^{-3}$

TABLE I

COMPUTATIONAL COMPLEXITY OF THE PROPOSED TECHNIQUES

\begin{tabular}{cc}
\hline \hline Technique & Number of Operations \\
\hline arctan-based ML (no bias correction) & $\mathrm{N}_{\mathrm{arg}}+(\mathrm{N}-1)$ \\
Bias correction for Linear Systems: Tech-1 & $8 \mathrm{~K}+\mathrm{N}_{\varsigma}+\mathrm{N}_{\mathrm{arg}}+\mathrm{N}+5$ \\
Bias correction for Linear Systems: Tech-2 & $(11 \mathrm{~K}+8) \mathrm{M}_{\beta}+\mathrm{N}_{\varsigma}+\mathrm{N}_{\mathrm{arg}}+(\mathrm{N}-1)$ \\
Particle Filter Method (N=1) & $10 \mathrm{M}+1+\mathrm{N}_{\mathrm{arg}}$ \\
arctan phase-detector-based D-PLL $(\mathrm{N}=1)$ & $\mathrm{N}_{\mathrm{arg}}+7$ \\
\hline
\end{tabular}

